\title{
Structure of cycles in minimal strong digraphs
}

\author{
Miguel Arcos-Argudo' , Jesús García-López , Luis M. Pozo-Coronado
}

Keywords:

Minimal strong digraphs

Structure of the cycles

Linear vertex

Strong component

\begin{abstract}
A B S T R A C T
This work shows a study about the structure of the cycles contained in a Minimal Strong Digraph (MSD). The structure of a given cycle is determined by the strongly connected components (or strong components, SCs) that appear after suppressing the arcs of the cycle. By this process and by the contraction of all SCs into single vertices we obtain a Hasse diagram from the MSD. Among other properties, we show that any SC conformed by more than one vertex (non trivial SC) has at least one linear vertex (a vertex with indegree and outdegree equal to 1) in the MSD (Theorem 1); that in the Hasse diagram at least one linear vertex exists for each non trivial maximal (resp. minimal) vertex (Theorem 2); that if an SC contains a number $\lambda$ of vertices of the cycle then it contains at least $\lambda$ linear vertices in the MSD (Theorem 3); and, finally, that given a cycle of length $q$ contained in the MSD, the number $\alpha$ of linear vertices contained in the MSD satisfies $\alpha \geq\lfloor(q+1) / 2\rfloor$ (Theorem 4 ).
\end{abstract}

\section{Introduction}

A minimal strong digraph (MSD) is a strong digraph in which the deletion of any arc yields a non strongly connected digraph. In [5] a compilation of properties of MSDs can be found, and in [6] an update of the catalog of properties is given, together with a comparative analysis between MSDs and non directed trees, from which a striking analogy between the two families of graphs emerges.

The configuration of cycles of an MSD determines, among many other things, the characteristic polynomial of its adjacency matrix and, therefore, it is very important for the spectral theory of this family of graphs [5,6]. It is also related to algorithmic problems that, restricted to MSDs, can present unexpected properties, given the strong analogy that exists between this family of graphs and trees [6]. In this way it is interesting to study the length $l$ of the longest cycle in an MSD, relate this parameter with the number of arcs and design an algorithm that calculates it quickly. Given the number $n$ of vertices and the number $m$ of arcs of the MSD, it is known [4] that

$$
\left\lceil\frac{m}{m-n+1}\right\rceil \leq l \leq 2 n-m
$$

and that these bounds are tight. The authors also relate the parameter $l$ to the number of linear vertices [1] of the MSD.

The structure of a cycle $C_{q}$ of length $q$ in an MSD $D$ is determined by the strongly connected components (strong components, SCs) of the digraph $D^{\prime}$ that appear after suppressing the arcs of the cycle. The contraction of these SCs generates 
an acyclic digraph that, having no transitive arcs because it comes from an MSD, is in fact a Hasse diagram. We analyze the properties of this Hasse diagram and its vertices (which are SCs of the digraph $D^{\prime}$ ).

The outline of the article is as follows: In Section 2 we set up notations and discuss some basic properties. In Section 3 we introduce some basic properties of the SCs of $D^{\prime}$ and present an algorithm that calculates the number of configurations of these SCs. In Section 4 we prove the results included in the abstract. Finally, in Section 5 we draw some conclusions.

\section{Notation and basic properties}

In this paper we use some concepts and basic results about graphs that are described below, in order to fix the notation $[2,5,6]$.

Let $D=(V, A)$ be a digraph. If $(u, v) \in A$ is an arc of $D$, we say that $u$ is the initial vertex and $v$ the final vertex of the arc and we denote the arc by $u v$. We shall consider only directed paths and directed cycles.

In a strongly connected digraph, the indegree and the outdegree of every vertex are greater than or equal to 1 . We shall say that $v$ is a linear vertex if it satisfies $d^{+}(v)=d^{-}(v)=1$.

An arc $u v$ in a digraph $D$ is transitive if there exists another $u v$-path disjoint to the arc $u v$. A digraph is called a minimal digraph if it has no transitive arcs.

The contraction of a subdigraph consists in the reduction of the subdigraph to a unique vertex. Note that the contraction of a cycle of length $q$ in an SD yields another SD. In such a process, $q-1$ vertices and $q$ arcs are eliminated.

A vertex $v$ on a digraph is called a cut point if the deletion of $v$ and all its incident arcs yields a disconnected digraph.

Some basic properties concerning MDSs can be found in $[2,3,5,6]$. We summarize some of them: In an MSD with $n$ vertices and $m$ arcs, $n \leq m \leq 2(n-1)$; the contraction of a cycle in an MSD preserves the minimality, that is, it produces another MSD; hence, if we contract a strongly connected subdigraph in a minimal digraph, the resulting digraph is also minimal; each MSD of order $n \geq 2$ has at least two linear vertices; in an MSD with exactly two linear vertices, each of them belongs to a unique cycle; furthermore, these cycles are $C_{2}$ or $C_{3}$. The next result will be explicitly used in some of our proofs.

Lemma 1 ([6]). If an MSD contains a cycle $C_{2}$, then the vertices on the cycle are linear vertices or cut points.

A strong component (SC) is a maximal strongly connected subdigraph. A trivial strong component is an SC that contains only one vertex. Note that every SC is a subdigraph induced by the set of its vertices.

Let $D$ be an MSD and $C_{q}$ a cycle contained in $D$. The digraph associated to $\left(D, C_{q}\right), D^{\prime}$, is the resultant digraph after suppressing in $D$ all arcs of $C_{q}$. The anchored SCs of $D^{\prime}$ are the SCs that contain vertices of $C_{q}$. The contraction of all SCs of $D^{\prime}$ yields an acyclic digraph $H$ with no transitive arcs. Hence, $H$ is minimal and can be seen as the Hasse diagram of a partial ordering. We call $H$ the Hasse diagram associated to $\left(D, C_{q}\right)$. A vertex on $H$ is minimal (resp. maximal) if its indegree (resp. outdegree) vanishes. Hence, minimal vertices on $H$ correspond to initial strong components of $D^{\prime}$, and maximal vertices on $H$ correspond to terminal strong components of $D$. We say that a maximal or minimal vertex of $H$ is non trivial if it has at least one incident arc.

\section{Basic properties of the anchored SCs of the digraph associated to $\left(D, C_{q}\right)$}

Let $D$ be an MSD, $C_{q}$ a cycle contained in $D$ and $D^{\prime}$ the digraph associated to $\left(D, C_{q}\right)$. Therefore, $D^{\prime}$ is not strongly connected and its SCs may contain vertices of $C$, even more than one. Note that any SC is a minimal digraph.

In this section we show some results obtained through an algorithm. This algorithm has been designed to exhaustively compute all possible SC configurations of the associated digraph $D^{\prime}$ for $2 \leq q \leq 19$. We have computed only non isomorphic SC configurations of the associated digraph $D^{\prime}$.

In order to compute all possible SC configurations we have considered all possible connections between vertices of $C_{q}$ and we have discarded the configurations in which at least one $\operatorname{arc}$ of $C_{q}$ has become transitive. Finally we have calculated and preserved only one canonical representative for each isomorphism class. below.

But, before passing to the description of the algorithms, we need some results on the SC structure that we demonstrate

Lemma 2. Let $D$ be an MSD, $C_{q}$ a cycle contained in $D$ and $D^{\prime}$ the digraph associated to $\left(D, C_{q}\right)$. An anchored $S C$ of $D^{\prime}$ cannot contain consecutive vertices of $C_{q}$. Hence, the number $\lambda$ of vertices of $C_{q}$ in a given anchored SC of $D^{\prime}$ satisfies $1 \leq \lambda \leq\left\lfloor\frac{q}{2}\right\rfloor$.

Proof. Suppose that an SC has two consecutive vertices $u$ and $v$. Then, the arc $u v$ would be transitive, contradicting the fact that $D$ is an MSD.

Let now $S$ be an $S C$ that contains a number $\lambda$ of vertices of $C_{q}$. Then, $S$ cannot contain consecutive vertices of $C_{q}$, and hence $\lambda \leq\left\lfloor\frac{q}{2}\right\rfloor$. The other inequality, $1 \leq \lambda$, is straightforward.

Definition 1. Let $D$ be an MSD, $C_{q}$ a cycle contained in $D$ and $D^{\prime}$ the digraph associated to $\left(D, C_{q}\right)$. We say that two anchored SCs of $D^{\prime}$ are cut if each one of them has two vertices in $C_{q}, u_{1}$ and $u_{2}$ in the first and $v_{1}$ and $v_{2}$ in the second, such that the order of these vertices in the cycle $C_{q}$ is $u_{1}, \ldots, v_{1}, \ldots, u_{2}, \ldots, v_{2}, \ldots, u_{1}$. 


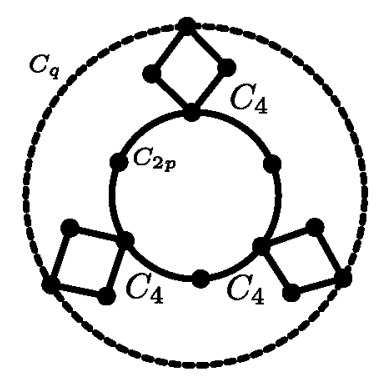

Fig. 1. Realization of a SC having $p$ vertices in $C_{q}$.

Lemma 3. Let $D$ be an MSD, $C_{q}$ a cycle contained in $D$ and $D^{\prime}$ the digraph associated to $\left(D, C_{q}\right)$. If two anchored SCs of $D^{\prime}$ are cut, they cannot have consecutive vertices in the cycle $C_{q}$.

Proof. Let $u$ and $v$ be vertices contained in $C_{q}$. Suppose that $u$ and $v$ are consecutive vertices of $C_{q}$ and are contained in distinct SCs of $D^{\prime}, u \in S C_{1}$ and $v \in S C_{2}$, that are cut. Then, the arc $u v$ would be transitive, contradicting the fact that $D$ is an MSD.

Now we present the algorithms that we have implemented. Each SC configuration is represented by an integer number array with length $q$, that we have called CompV and should verify the following properties:

(1) CompV $[k]$ represents the SC to which the vertex $k$ belongs.

(2) $0 \leq$ CompV $[k]<q$ for all $0 \leq k<q$ (the maximum number of components is $q$ ).

(3) CompV $[k] \neq \operatorname{CompV}[(k+1) \bmod q]$ for all $0 \leq k<q$ (by Lemma 2).

(4) CompV $[0]=0$ (given a SC configuration, there is always a numbering of the SCs that verifies this).

(5) CompV $[k] \leq 1+\max \{\operatorname{CompV}[j] \mid 0 \leq j<k\}$ for all $0<k<q$ (given a SC configuration, there is always a numbering of the SCs that verifies this).

The first algorithm implements the "Next" function that, starting in the initial configuration, generates all the SC configurations. Every time it is executed it calculates the following configuration to the current one. It generates all SC configurations in lexicographical order of the CompV array. The initial and final configurations are as follows:

(1) Initial: $[0,1,0, \ldots, 0,1]$, if $q$ is even, or $[0,1,0,1, \ldots, 0,1,2]$, if $q$ is odd.

(2) Final: $[0,1,2, \ldots, q-2, q-1]$.

\section{Algorithm 1. Next function}

(i) Locate (looking for from $(q-1)$ to 0 ) the first index $k$ that allows to increase the value of CompV $[k]$. The index $k$ is the first that verifies

$$
\operatorname{CompV}[k] \leq \max \{\operatorname{CompV}[j] \mid 0 \leq j<k\} .
$$

(ii) If $k=0$ return NULL (There are no more configurations. The current and final configurations are the same).

(iii) Assign a new value to CompV $[k], c^{\circ} p_{k}$ :

$$
\operatorname{comp}_{k}= \begin{cases}\operatorname{CompV}[k]+1 & \text { if CompV }[k-1] \neq \operatorname{CompV}[k]+1 \\ \operatorname{CompV}[k]+2 & \text { if CompV }[k-1]=\operatorname{CompV}[k]+1\end{cases}
$$

(iv) Assign values to CompV[j] for $k<j<q$ :

$$
\operatorname{CompV}[j]= \begin{cases}0 & \text { if } j \neq q-1 \text { and } \operatorname{CompV}[j-1] \neq 0 \\ 1 & \text { if } \operatorname{CompV}[j-1]=0 \\ 1 & \text { if } j=q-1 \text { and } \operatorname{CompV}[j-1] \neq 1 \\ 2 & \text { if } j=q-1 \text { and } \operatorname{CompV}[j-1]=1\end{cases}
$$

(v) Return CompV.

Algorithm 1 generates all the SC configurations that verify Lemma 2, i.e., configurations in which no SC contains consecutive vertices of the cycle $C_{q}$. According to Lemma 3, configurations containing SCs that are cut and have consecutive vertices of $C_{q}$ must be discarded. It is easy to prove that every SC configuration verifying Lemmas 2 and 3 is feasible as MSD. Fig. 1 shows a way to build the SCs.

To preserve only a canonical representative we have implemented the "Canonical" function, that considers the $q$ arrays that are obtained when fixing each one of the positions of the circular array CompV as origin. The canonical representative will be the least of them, using lexicographical order. 
Table 1

Number of configurations of SCs of the digraph associated to $\left(D, C_{q}\right)$.

\begin{tabular}{rclc}
\hline \multicolumn{1}{c}{$q$} & Num. of SCs & $q$ & Num. of SCs \\
\hline 2 & 1 & 11 & 162 \\
3 & 1 & 12 & 427 \\
4 & 2 & 13 & 1016 \\
5 & 2 & 14 & 2836 \\
6 & 5 & 15 & 7432 \\
7 & 6 & 16 & 20579 \\
8 & 16 & 17 & 52622 \\
9 & 28 & 18 & 159172 \\
10 & 43 & 19 & 449390 \\
\hline
\end{tabular}

Algorithm 2. Canonical function

(i) Copy CompV in CompC (CompC is going to be the canonical array).

(ii) For every $0<k<q$ : $\circ$ Calculate $A u x=[\operatorname{CompV}[k]$, ldots, $\operatorname{CompV}[k+q-1]]($ indices $\bmod q)$.

- Renumbering the SCs of Aux so that CompV properties hold:

- Initialize $\operatorname{ReN}[j]=0$ for $0 \leq j<q$ (to indicate whether vertex $j$ is renumbered or not).

- NewComp $=0$.

- For $j=0$ to $j=q-1$ :

$\star$ If $R e N[j]=0$ (the vertex $j$ is not renumbered), then:

$*$ Comp $=A u x[j]$.

$*$ For $j \leq k<q$ do: if $A u x[k]=$ Comp and $\operatorname{Re} N[k]=0$ :

$\diamond A u x[k]=$ NewComp and ReN $[k]=1$.

$*$ NewComp $=$ NewComp +1 .

(iii) Return CompC.

o If $A u x<C o m p C$ in lexicographical order, copy Aux in CompC.

Table 1 shows the obtained results. Note how the number of SC configurations of the associated digraph $D^{\prime}$ increases exponentially as $q$ grows.

From the experimental data, we conjecture that the number of anchored SCs of $D^{\prime}$ has $\lfloor(q+3) / 2\rfloor$ as a lower bound.

\section{Properties of the $S C s$ of the digraph associated to $\left(D, C_{q}\right)$}

Throughout this section, we keep using the notations introduced in the first sentence of Section 3, adding the Hasse diagram $H$. If $S$ is a SC of a digraph $D^{\prime}$, we shall denote by s the corresponding vertex of $H$. Now, we state and prove our main results.

Remark 1. Let $D$ be an MSD, $C_{q}$ a cycle contained in $D$ and $D^{\prime}$ and $H$ the digraph and the Hasse diagram respectively associated to $\left(D, C_{q}\right)$. Let $s_{1}, \ldots, s_{j}$ be a path in $H$ and $u$ and $v$ vertices of $D$ such that $u \in S_{1}$ and $v \in S_{j}$. Then it is obvious that there exists a $u v$-path in $D$ passing by $S_{1}, \ldots, S_{j-1}$ and $S_{j}$.

Lemma 4. Let $D$ be an MSD, $C_{q}$ a cycle contained in $D$ and $D^{\prime}$ and $H$ the digraph and the Hasse diagram respectively associated to $\left(D, C_{q}\right)$. Then a cycle with no linear vertices in $D$ cannot be an SC of $D^{\prime}$.

Proof. First, we state that an SC $S$ corresponding to a maximal or minimal vertex of $H$ contains at least one vertex of $C_{q}$.

In fact, if $S$ is a maximal (resp. minimal) vertex in $H$, there are no output (resp. input) arcs from any vertex of $S$ to any vertex of any other SC (resp. to any vertex of $S$ from any vertex of any other SC). There is at least one SC different from $S$, because $D^{\prime}$ is not strongly connected. The strong connection of $D$ implies that there exists an arc leaving (resp. entering) $S$. This arc must belong to $C_{q}$ and consequently $S$ contains at least one vertex of $C_{q}$.

Now, let $S$ be an SC and let $u, v \in S$ be two distinct vertices such that there exist arcs $u a$ and $b v$ in $D$ with $a, b \notin S$. Then there exists in $D$ a $u v$-path that does not contain arcs of $S$.

Indeed, let us start in $s_{1}=s$ and walk through $H$, leaving $s_{1}$ through the arc $s_{1} s_{2}$ (where $S_{2}$ is the SC containing $a$ ), until we find a maximal vertex on $H: s_{1}, s_{2}, \ldots, s_{j}$. According to the paragraph above, in $S_{j}$ there must exist at least one vertex of $C_{q}$, say $w$. Then by Remark 1 there exists a $u w$-path in $D$. Now we start again in $s_{1}^{\prime}=s$ and we walk through $H$ in opposite direction, leaving $s$ through the $\operatorname{arc} s_{2}^{\prime} s_{1}^{\prime}$ (where $S_{2}^{\prime}$ is the SC containing $b$ ), until we find a minimal vertex of $H: s_{k}^{\prime}, s_{k-1}^{\prime}, \ldots, s_{2}^{\prime}, s_{1}^{\prime}$. Again, $S_{k}^{\prime}$ must contain at least one vertex of $C_{q}$, say $t$. Then we obtain a $t v$-path in $D$ (applying Remark 1 to the path $s_{k}^{\prime}, \ldots, s_{2}^{\prime}, s_{1}^{\prime}$ ). It is obvious that there exists a $w t$-path in $C_{q}$. Therefore there exists a $u v$-path in $D$ (obtained by the concatenation of the $u w$-path, the $w t$-path and the $t v$-path) that does not use arcs of $S$.

We prove now that a cycle $C$ with no linear vertices in $D$ cannot be an SC of $D^{\prime}$. Let a cycle $C$ be an SC of $D^{\prime}$ and let us consider by contradiction that it does not contain any linear vertex on $D$. In that case, each vertex of $C$ should either have 
an input arc from or an output arc to an SC different from $C$, or else be contained in $C_{q}$. Hence $C$ must contain at least two consecutive vertices $u$ and $v$ such that: 1$) u$ belongs to $C_{q}$ or there is an arc $u a$ in $D$ with $a \notin C$; and 2) $v$ belongs to $C_{q}$ or there is an $\operatorname{arc} b v$ in $D$ with $b \notin C$.

It is thus clear, as we have shown above, that in $D$ there exists a $u v$-path that does not contain arcs of $C$. As a consequence, the arc $u v$ contained in $C$ would be transitive in $D$. This fact contradicts the minimality of $D$ and completes the proof.

Theorem 1. Let $D$ be an MSD, $C_{q}$ a cycle contained in $D$ and $D^{\prime}$ and $H$ the digraph and the Hasse diagram respectively associated to $\left(D, C_{q}\right)$. Then, any non trivial $S C$ of $D^{\prime}$ with at most one vertex of $C_{q}$ has at least one linear vertex in $D$.

Proof. Let $S$ be a non trivial SC of $D^{\prime}$ with at most one vertex of $C_{q}$. Let $k$ be the number of vertices in $S$. We prove the result by induction on $k$.

Base step: If $k=2$ then $S$ is the cycle $C_{2}$ and Lemma 4 implies the result.

Induction step: Let $S$ be an SC conformed by $k+1$ vertices $(k \geq 2)$ which has at most one vertex of $C_{q}$, and suppose by induction hypothesis that any SC with at most $k$ vertices which has at most one vertex of $C_{q}$ contains at least one linear vertex in $D$.

If $S$ is the cycle $C_{k+1}$ then Lemma 4 implies the result.

Suppose that $S \neq C_{k+1}$. Then $S$ contains a cycle $C$ of length $p, 2 \leq p \leq k$. Assume that $C$ does not contain any linear vertex in $D$ (otherwise there is nothing to prove). $S$ must contain at least one vertex that is not contained in $C$. The contraction of $C$ in a unique vertex, say $z$, produces another MSD $\bar{D}$ that maintains the cycle $C_{q}$ (because $S$ contains at most one vertex of this cycle), and another SC $\bar{S}$ belonging to the digraph $\bar{D}^{\prime}$ associated to $\left(\bar{D}, C_{q}\right)$. The number of vertices of $\bar{S}$ is equal to $k-p+2$, where $2 \leq k-p+2 \leq k$. Hence, by induction hypothesis, $\bar{S}$ must contain at least one linear vertex in $\bar{D}$, say $u$. Since $z$ represents $C$, which has no linear vertices, it must be $u \neq z$ because, in $\bar{D}$, degree $(z) \geq 3$ (if $k=2$, each vertex of $C_{2}$ has degree $\geq 4$, since, by Lemma 1 , both of them are cut points). Therefore, $u$ is a linear vertex in $D$.

The proof is thus complete.

Remark 2. Let $D$ be an MSD, $C_{q}$ a cycle contained in $D$ and $D^{\prime}$ and $H$ the digraph and the Hasse diagram respectively associated to $\left(D, C_{q}\right)$. Let $u v$ be an arc of $H$. Then the corresponding SCs of these vertices $u, v$ of $H$ cannot simultaneously contain vertices of $C_{q}$.

Lemma 5. Let $D$ be an MSD, $C_{q}$ a cycle contained in $D$ and $D$ and $H$ the digraph and the Hasse diagram, respectively associated to $\left(D, C_{q}\right)$. Then $H$ has at least one linear vertex for each path joining a minimal vertex to a maximal vertex.

Proof. Let $u_{0}, \ldots, u_{j}$ be a $u_{0} u_{j}$-path in $H$ joining a minimal vertex $u_{0}$ with a maximal vertex $u_{j}$.

We begin by remarking that $j \geq 2$. In fact, suppose there is an arc in $H$ joining the minimal vertex $u_{0}$ to the maximal vertex $u_{1}$. In the proof of Lemma 4 we have shown that both $U_{0}$ and $U_{1}$ must contain vertices of $C_{q}$, which is impossible by Remark 2.

Let us now suppose, by contradiction, that there are no linear vertices in the $u_{0} u_{j}$-path. Hence, $d^{-}\left(u_{1}\right)=1$ or else the arc $u_{0} u_{1}$ would be transitive in $D$. In fact, if $v_{1}$ is the minimal vertex reached by walking in reverse direction from $u_{1}$ using an $\operatorname{arc} u_{1}^{\prime} u_{1}$ different from $u_{0} u_{1}$ (such an arc exists because of $d^{-}\left(u_{1}\right)>1$ ) then a $u_{0} u_{1}$-path (not containing the $\operatorname{arc} u_{0} u_{1}$ ) can be obtained by concatenation of a $u_{0} v_{1}$-path with the $v_{1} u_{1}$-path.

Then, $d^{+}\left(u_{1}\right)>1$, since $d^{-}\left(u_{1}\right)=1$ and we are assuming that $u_{1}$ is not linear. Let $u_{1}^{\prime \prime} \neq u_{2}$ be the vertex defined by the corresponding arc $u_{1} u_{1}^{\prime \prime} \in D$.

Now the following result will be proved for all $u_{i}, 2 \leq i<j: d^{-}\left(u_{i}\right)=1$ and there is an arc $u_{i} u_{i}^{\prime \prime}$ with $u_{i}^{\prime \prime} \neq u_{i+1}$. To show this, the following reasoning is applied iteratively for each vertex, starting from $u_{2}$. First, we remark that $d^{-}\left(u_{i}\right)=1$. Otherwise, the $\operatorname{arc} u_{i-1} u_{i}$ would be transitive in $D$, because an $u_{i-1} u_{i}$-path would exist, not containing the $\operatorname{arc} u_{i-1} u_{i}$. In fact, let $v_{i}$ be the minimal vertex that is reached walking in reverse direction from $u_{i}$ starting with an arc $u_{i} u_{i}$ different from $u_{i-1} u_{i}$ (such an arc exists, since $d^{-}\left(u_{i}\right)>1$ ). Let $w_{i-1}$ be the maximal vertex reached walking from $u_{i-1}$ starting with the arc $u_{i-1} u_{i-1}^{\prime \prime}$. Then an $u_{i-1} u_{i}$-path would be obtained by concatenation of the $u_{i-1} w_{i-1}$-path with the $w_{i-1} v_{i}$-path and with the $v_{i} u_{i}$-path.

Besides, $d^{+}\left(u_{i}\right)>1$ also holds, because $d^{-}\left(u_{i}\right)=1$ and, by hypothesis, $u_{i}$ is not a linear vertex. Let $u_{i}^{\prime \prime} \neq u_{i+1}$ be the vertex defined by the arc $u_{i} u_{i}^{\prime \prime}$ belonging to $H$.

Finally, let us show that the arc $u_{j-1} u_{j}$ is transitive. In fact, let $w_{j-1}$ be the maximal vertex reached walking from $u_{j-1}$, starting with the arc $u_{j-1} u_{j-1}^{\prime \prime}$. The $u_{j-1} u_{j}$-path obtained by concatenation of the $u_{j-1} w_{j-1}$-path and the $w_{j-1} u_{j}$-path proves that $u_{j-1} u_{j}$ is transitive. This fact contradicts the minimality of $D$ and concludes the proof (see Fig. 2).

Note that the linear vertices resulting from the lemma above cannot be the first nor the last vertex of the original path.

Let $D$ be an MSD, $C_{q}$ a cycle contained in $D$, and $D^{\prime}$ and $H$ the digraph and the Hasse diagram respectively associated to $\left(D, C_{q}\right)$. We call pseudominimal (resp. pseudomaximal) vertex of $H$ to any vertex corresponding to an anchored SC with outdegree (resp. indegree) greater than 0 . Note that a pseudominimal vertex which is not minimal has to be also pseudomaximal. Note also that an anchored SC must be either pseudominimal, pseudomaximal, or trivial. Finally, we remark that the proof of Lemma 5 is also valid for paths whose endpoints are pseudominimal or pseudomaximal vertices. 


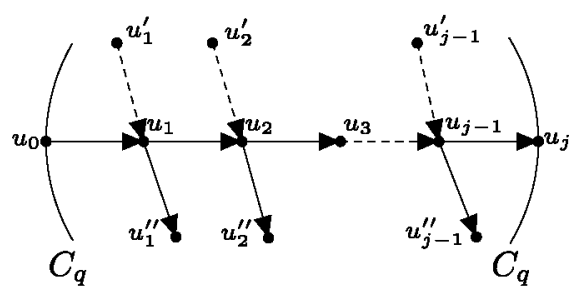

Fig. 2. Illustration of Lemma 5.

Theorem 2. Let $D$ be an MSD, $C_{q}$ a cycle contained in $D$ and $D^{\prime}$ and $H$ the digraph and the Hasse diagram respectively associated to $\left(D, C_{q}\right)$. Then, the number of linear vertices of $H$ is greater than or equal to the number of pseudominimal (resp. pseudomaximal) vertices of $H$.

Proof. Let $u_{1}, \ldots, u_{k}$ be the pseudominimal vertices of $H$. For all $u_{i}, 1 \leq i \leq k$, consider a $u_{i} v_{i}$-path from $u_{i}$ to a pseudomaximal vertex $v_{i}$ of $H$. Applying Lemma 5 , let $w_{i}$ be the first linear vertex that appears in this path. By the construction used in the proof of Lemma 5 , the vertices $w_{1}, \ldots, w_{k}$ can be shown to be different to each other. In fact, given a $u_{i}, 1 \leq i \leq k$, the vertices of the $u_{i} w_{i}$-path cannot belong to any of the paths $u_{j} v_{j}$-path $(1 \leq j \leq k, j \neq i)$ because the indegree of each vertex is 1 or $0\left(u_{i}\right)$. This fact implies the result in the pseudominimal vertices case. An analog demonstration of Lemma 5 , and the previous result, but starting in the pseudomaximal vertices, concludes the proof.

Remark 3. Lemma 5 also holds if we consider paths with end vertices in $C_{q}$ and contained in SCs in which all cycles include vertices of $C_{q}$.

Theorem 3. Let $D$ be an MSD, $C_{q}$ a cycle contained in $D$ and $D^{\prime}$ the digraph associated to $\left(D, C_{q}\right)$. An SC $S$ of $D^{\prime}$ that contains a number $\lambda>1$ of vertices of $C_{q}$ has at least $\lambda$ linear vertices in $D$.

Proof. Let $u_{0}, \ldots, u_{\lambda-1}$ be the $\lambda$ vertices of $C_{q}$ belonging to $S$. Let $\bar{D}$ be the MSD obtained from $D$ after iterative contraction of the cycles that do not include vertices of $C_{q}$. For each vertex $u_{i}, 0 \leq i<\lambda$, consider a $u_{i} u_{i+1}$-path ( $i$ module $\left.\lambda\right)$ in $\bar{D}$. Remark 3 proves that each $u_{i} u_{i+1}$-path has at least a linear vertex in $\bar{D}$ and that, if $w_{i}$ is the first linear vertex in the $u_{i} u_{i+1}$-path, the indegree of all vertices of the $u_{i} w_{i}$-path (not considering $u_{i}$ ) is equal to 1 . This implies, in turn, that the vertices $w_{0}, \ldots, w_{\lambda-1}$ are all different.

To get back the configuration of $D$ we can iteratively expand, in reverse order, the vertices obtained as a result of contraction of the cycles. Throughout this process we update the linear vertices $w_{i}, 0 \leq i<\lambda$, in the following way. Let $x$ be the vertex to expand, and $C_{x}$ the generated cycle as result of the expansion. If $x$ is not a linear vertex, there is nothing to update. However, if $x$ is a linear vertex, it is necessary to prove that a new linear vertex exists in $C_{x}$. Let $x_{1}$ and $x_{2}$ be the vertices of $C_{x}$ that define the input arc and the output arc respectively in $C_{x}$ (there are exactly 2 because $x$ is a linear vertex). If the length of $C_{x}$ is greater than or equal to 3 , or the length is 2 and $x_{1}=x_{2}$, then $C_{x}$ has a linear vertex. It remains to analyze the case where $C_{x}$ has length 2 and $x_{1} \neq x_{2}$. In this case, we have that $C_{x}=C_{2}$, neither of the two vertices of $C_{x}$ is linear, because they both have a total degree greater than or equal to 3 , and neither of them is a cut point because $x_{1}$ and $x_{2}$ are connected by a path, coming from any cycle that passed through $x$ before the expansion of $C_{x}$. This situation contradicts Lemma 1. Therefore, this case is not possible and the proof concludes.

Theorem 4. Let $D$ be an MSD and $C_{q}$ a cycle contained in $D$. Then, the number of linear vertices of $D$ is greater than or equal to $\left\lfloor\frac{q+1}{2}\right\rfloor$.

Proof. Let $D^{\prime}$ and $H$ be the digraph and the Hasse diagram, respectively, associated to $\left(D, C_{q}\right)$. Let $n_{1}$ be the number of vertices of $C_{q}$ that constitute trivial SCs, and that are not both maximal and minimal. Let $\alpha$ be the number of linear vertices of $D$. Each one of the vertices of $C_{q}$ not counted in $n_{1}$ belongs to a non trivial SC and, by Theorems 1 and 3 , either contributes with a linear vertex or is itself a linear vertex in $D$ (if the vertex is a trivial SC which is both maximal and minimal). Besides, the maximum between the number of pseudomaximals and the number of pseudominimals of $D^{\prime}$ is greater than or equal to $\left\lceil\frac{n_{1}}{2}\right\rceil$. Therefore, by applying Theorem 2 , we obtain

$$
\alpha \geq q-n_{1}+\left\lceil\frac{n_{1}}{2}\right\rceil=q-\left\lfloor\frac{n_{1}}{2}\right\rfloor
$$

The minimum of the right term of the previous inequality is reached when $n_{1}=q$ and hence

$$
\alpha \geq q-\left\lfloor\frac{q}{2}\right\rfloor=\left\lceil\frac{q}{2}\right\rceil=\left\lfloor\frac{q+1}{2}\right\rfloor
$$




\section{Conclusions}

The deletion of the arcs of a cycle $C_{q}$ in an MSD and the structure of the SCs in the obtained digraph $D^{\prime}$ gives an insight of the possible ways in which a cycle can be imbedded into an MSD. Some interesting properties of MSDs can be derived from this procedure. In fact, it has allowed us to prove that an MSD containing a $q$-cycle must have at least $\lfloor(q+1) / 2\rfloor$ linear vertices. We think that further work on this direction will lead to a deeper understanding of the cycle structure of MSDs, and hence of the spectral properties of this family of digraphs.

\section{Acknowledgments}

We want to express our gratitude to the unknown referees, whose suggestions and careful reading of our manuscript have greatly improved the final result.

\section{References}

[1] M. Arcos-Argudo, J. García-López, L.M. Pozo-Coronado, Estructura de ciclos en digrafos fuertemente conexos minimales, in: 10th Andalusian Meeting on Discrete Mathematics, La Línea de la Concepción, Cádiz, Spain, July 10-112017.

[2] J. Bang-Jensen, G. Gutin, Digraphs: Theory, Algorithms and Applications, second ed., Springer, London, 2009.

[3] C. Berge, Graphes, North-Holland, Amsterdam, 1991.

[4] Z.B. Chen, F.J. Zhang, Bounds of the longest directed cycle length for minimal strong digraphs, Discrete Math. 68 (1988) 9-13.

[5] J. García-López, C. Marijuán, Minimal strong digraphs, Discrete Math. 312 (4) (2012) 737-744.

[6] J. García-López, C. Marijuân, L.M. Pozo-Coronado, Structural properties of minimal strong digraphs versus trees, Linear Algebra Appl. 540 (2018) $203-220$ 